\title{
Quantization of Equations of Motion
}

D. Kochan

The Classical Newton-Lagrange equations of motion represent the fundamental physical law of mechanics. Their traditional Lagrangian and/or Hamiltonian precursors when available are essential in the context of quantization. However, there are situations that lack Lagrangian and/or Hamiltonian settings. This paper discusses a description of classical dynamics and presents some irresponsible speculations about its quantization by introducing a certain canonical two-form $\Omega$. By its construction $\Omega$ embodies kinetic energy and forces acting within the system (not their potential). A new type of variational principle employing differential two-form $\Omega$ is introduced. Variation is performed over "umbilical surfaces " instead of system histories. It provides correct Newton-Lagrange equations of motion. The quantization is inspired by the Feynman path integral approach. The quintessence is to rearrange it into an "umbilical world-sheet" functional integral in accordance with the proposed variational principle. In the case of potential-generated forces, the new approach reduces to the standard quantum mechanics. As an example, Quantum Mechanics with friction is analyzed in detail.

Keywords: quantization of dissipative systems, umbilical strings, path vs. surface integral.

Dedicated to Tulka.

\section{Introduction and motivation}

A detailed understanding and description of quantal and classical phenomena has attracted the attention of many mathematicians and physicists for a long time. Quantum and classical mechanics are the best elaborated, understood and examined parts of physics. Their mathematical setting is concentrated around powerful artillery, which includes differential geometry, spectral calculus, functional analyses, group and representation theory, (co)homology techniques, and so on.

The problem of how to get directly from classical dynamics, represented by the system of second-order differential equations of the Newton-Lagrange type,

$$
\ddot{x}^{i}=\mathbb{F}^{i}(x, \dot{x}, t)
$$

to the corresponding quantum dynamics was articulated by Feynman, see Freeman Dyson's editorial comment [1]. Standard approaches are based on canonical quantization (Heisenberg-like and/or Schrödinger-like equations) or on the Feynman path integral technique. All of these procedures require in some sense Lagrangian $\mathbb{L}=\mathbb{T}-\mathbb{U}$ and/or Hamiltonian $\mathbb{H}=\mathbb{T}+\mathbb{U}$ functions, such that

$\ddot{x}^{i}=\mathbb{F}^{i}(x, \dot{x}, t) \Leftrightarrow \frac{\delta \mathbb{L}}{\delta x^{i}}=0 \quad$ and $/$ or $\quad \frac{\delta \mathbb{H}}{\delta x^{i}}=0$.

Here we have imprecisely denoted the functional derivatives of the underlying classical variational principles by $\frac{\delta \mathbb{L}}{\delta x}$ and/or $\frac{\delta \mathbb{H}}{\delta x}$. The fact that kinetic energy $\mathbb{T}$ is a time-independent quadratic form in velocities and/or the corresponding momenta is absolutely crucial for the classical and quantal descriptions. It provides an intimate connection with the universal mechanical property known as inertia. The question of, whether there exists a Lagrangian and/or Hamiltonian for the given set of forces for the initial Newton-Lagrange system has been studied by many authors, for more detail see for example [2-4]. This problem is known as the inverse problem of the calculus of variation. In many applications, requirements such as the quadraticity and time-independence of $\mathbb{T}$ are suppressed. For example, for a one-dimensional free particle driven by friction proportional to the actual velocity, one can assign to any real number $\alpha$ the Lagrangian function $\mathbb{L}_{\alpha}$ as follows:

$\mathbb{L}_{\alpha}(x, \dot{x}, t)= \begin{cases}\frac{\dot{x}^{\alpha+1}}{\alpha(\alpha+1)} \mathrm{e}^{\alpha t}, & \text { for } \alpha \in R /\{-1,0\} \\ \dot{x} \ln \dot{x}-\dot{x}-x, & \text { for } \alpha=0 \\ -\mathrm{e}^{-t} \ln \dot{x}, & \text { for } \alpha=-1\end{cases}$

then $\frac{\delta \mathbb{L}_{\alpha}}{\delta x}=0 \quad \Leftrightarrow \quad \ddot{x}=-\dot{x}$.

No two of the Lagrangians listed above are equivalent, i.e. $\mathbb{L}_{\alpha}-$ const $\mathbb{L}_{\alpha^{\prime}} \neq \frac{\mathrm{d}}{\mathrm{d} t} f(x, t)$. This simple observation in the simplest example under consideration has very strong physical consequences in general. The non-equivalent Lagrangians lead to non-equivalent quantum mechanics (this is unrelated to the problem of ordering), i.e. the transition amplitudes computed according to them are different, though their classical limit is the same. This problem is called quantization ambiguity. It is possible to say which Lagrangian provides the "genuine" quantum mechanics only after performing a suitable experiment, but the role of quadraticity and time independency of $\mathbb{T}$ will surely be essential there.

The aim of this paper is to try to find an answer to the Feynman problem, and, at the same time, to provide a geometrical picture of classical and quantum mechanics for physical systems, where a proper Lagrangian and/or Hamiltonian description is missing. The central object in our approach is a certain canonical two-form $\Omega$, which is defined in an extended tangent bundle. Its main properties are narrowly studied in section 2 . The differential two-form $\Omega$ serves as a guide for a new type of variational principle. In section 3 we introduce the notion of an "umbilical world-sheet." It generalizes the concept of the history of the system and there- 
fore it becomes important in the context of quantization. Variation uncovers the desired classical trajectory and, as a bonus, also some kind of minimal surface. In section 4 we will see how "umbilical strings" can be used to rearrange the Feynman integral over the histories of the system to the surface functional integral. String formulation has the big advantage that it concerns components of the forces rather than their potential. In section 5 we are able to compute the transition probability amplitude for a quantum system with friction explicitly performing the surface functional integration. For potential-generated forces, the "umbilical world-sheet" approach reduces to standard quantum mechanics.

This paper follows up ideas presented in my previous work. Many facts briefly mentioned here can be consulted in detail in $[5,6]$.

\section{Lagrangian mechanics and the two-form $\Omega$}

The physical content of classical mechanics is represented by Newton's dynamical law. Its formulation in general curvilinear coordinates (after resolving e.g. the initial holonomic constraints) coincides with the Lagrange equations [7-10]:

$\frac{\mathrm{d}}{\mathrm{d} t}\left(\frac{\partial \mathbb{T}}{\partial \dot{q}^{i}}\right)-\frac{\partial \mathbb{T}}{\partial q^{i}}=\mathbb{Q}_{i}$,

$i=1, \ldots, n$ (= number of degrees of freedom).

Here, $\mathbb{T}(q, \dot{q}, t)=\frac{1}{2} T_{a b}(q, t) \dot{q}^{a} \dot{q}^{b}$ is the kinetic energy of the system and $\mathbb{Q}_{i}(q, \dot{q}, t)=\mathrm{F}_{\mu} \frac{\partial r^{\mu}}{\partial q^{i}}$ is the $i$-th component of a generalized force. In the special case when forces are potential-generated

$$
\mathbb{Q}_{i}=-\frac{\partial \mathbb{U}}{\partial q^{i}}+\frac{\mathrm{d}}{\mathrm{d} t}\left(\frac{\partial \mathbb{U}}{\partial \dot{q}^{i}}\right)
$$

one can introduce the Lagrangian function $\mathbb{L}=\mathbb{T}-\mathbb{U}$ and write down the celebrated Euler-Lagrange equations. Generalized coordinates $\left\{q^{i}\right\}$ cover some open patch of the configuration space ( $n$-dimensional manifold) $M$.

Let us trace out the importance of kinetic energy $\mathbb{T}$ in a geometrical description of mechanics. Components $T_{a b}$ can be interpreted as some Riemannian metric on $M$. Here the fact that $\mathbb{T}$ is the quadratic function in the generalized velocities is absolutely crucial. After introducing a RLC-connection $\nabla$ for such a metric (kinetic energy), one can rewrite (1) in the closed form:

$$
\frac{\nabla p_{i}}{\mathrm{~d} t}=\mathbb{Q}_{i}, \text { where } p_{i}:=\frac{\partial \mathbb{T}}{\partial \dot{q}^{i}} .
$$

We immediately realize that for the free case the system evolves along a geodetic specified by the Riemannian connection $\nabla$. This, hopefully, sheds some light on the phenomenon called inertia.

In the Lagrangian picture, the space of all physical states is the set of all admissible initial conditions for the differential system (1). The initial condition specified at time $t_{0}$ by the generalized position $q\left(t_{0}\right)=q_{0}$ and velocity $\dot{q}\left(t_{0}\right)=v_{0}$, defines a point $\left(q_{0}, v_{0}, t_{0}\right)$ in an extended tangent bundle $T M \times \mathbb{R}$. For the extended tangent bundle coordinates we will use the $(2 n+1)$-tuple $\left\{q^{1}, \ldots, q^{n}, v^{1}, \ldots, v^{n}, t\right\}$.

Let us express from (1) generalized accelerations as functions of the remaining entries:

$$
\begin{aligned}
\ddot{q}^{i}=f^{i}(q, \dot{q}, t, \mathbb{Q}) \equiv & \left(\frac{\partial^{2} \mathbb{T}}{\partial \dot{q}^{i} \partial \dot{q}^{a}}\right)^{-1} \\
& \left\{\mathbb{Q}_{a}(q, \dot{q}, t)+\frac{\partial \mathbb{T}}{\partial q^{a}}-\frac{\partial^{2} \mathbb{T}}{\partial \dot{q}^{a} \partial q^{b}} \dot{q}^{b}-\frac{\partial^{2} \mathbb{T}}{\partial \dot{q}^{a} \partial t}\right\} .
\end{aligned}
$$

When identifying $\dot{q}^{i}$ with $v^{i}$, we get instead of (1) the system of $(2 n+1)$ first-order differential equations in the extended tangent bundle:

$$
\dot{q}^{i}=v^{i}, \quad \dot{v}^{i}=f^{i}(q, v, t, \mathbb{Q}), \quad \dot{t}=1 .
$$

The system above can be interpreted as a coordinate expression of a vector field on $T M \times \mathbb{R}$; down-to-earth, according to (3) one can assign to any physical state $(q, v, t)$ a tangent vector

$$
\begin{aligned}
\left.\Gamma\right|_{(q, v, t)}= & \left.\partial_{t}\right|_{(q, v, t)}+\left.v^{i} \partial_{q^{i}}\right|_{(q, v, t)} \\
& +\left.f^{i}(q, v, t, \mathbb{Q}) \partial_{v^{i}}\right|_{(q, v, t)} \in T_{(q, v, t)}(T M \times \mathbb{R}) .
\end{aligned}
$$

The time evolution is represented by a curve in the extended tangent bundle (see Fig. 1)

$\gamma: \mathbb{R}[\tau] \rightarrow T M \times \mathbb{R}, \tau \mapsto \gamma(\tau) \equiv q=q(\tau), v=v(\tau)$, such that $\left.\frac{\mathrm{d}}{\mathrm{d} t} \gamma(\tau)\right|_{\gamma(\tau)}=\left.\Gamma\right|_{\gamma(\tau)}$.

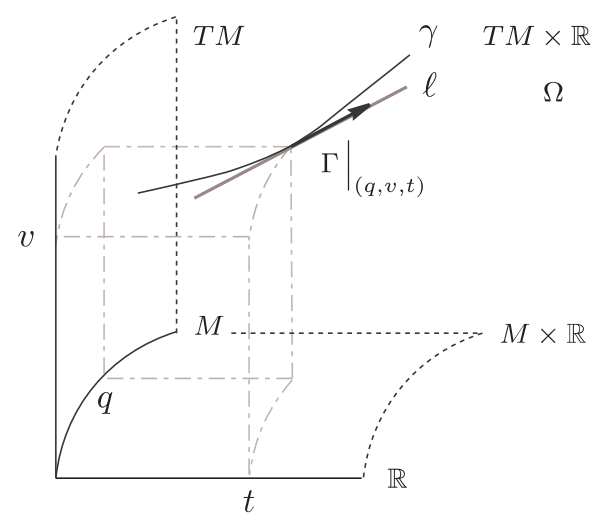

Fig. 1: Time evolution in the Lagrangian picture is bedded in the extended tangent bundle. At each physical state $(q, v, t)$ there is a uniquely prescribed vector $\left.\Gamma\right|_{(q, v, t)}$, which defines the dynamics. Following its integral curves, the complete time evolution is recovered

We have just observed that classical dynamics is determined by the extended tangent bundle vector field $\Gamma$. Having the function $\mathbb{T}(q, v, t)$ and the components of the generalized force $\mathbb{Q}(q, v, t)$ we can establish the two-form

$\Omega:=\left\{\mathbb{Q}_{i} \mathrm{~d} q^{i}\right\} \wedge \mathrm{d} t+\mathrm{d}\left\{\mathbb{L} \mathrm{d} t+\left(\partial_{v}{ }^{i} \mathbb{L}\right)\left\{\mathrm{d} q^{i}-v^{i} \mathrm{~d} t\right\}\right\}$. 
Its main properties can be summarized as follows:

- it is a differential two-form on the extended tangent bundle

- for any point $(q, v, t)$, it provides the linear map

$\downarrow: T_{(q, v, t)}(T M \times \mathbb{R}) \rightarrow T_{(q, v, t)}^{*}(T M \times \mathbb{R})$,

$w \mapsto \alpha:=\Omega(w, \cdot) \equiv w, \Omega$

- if $\mathbb{T}$ is nonsingular $\left\{\Leftrightarrow \partial_{v^{i} v^{a}}^{2}(\mathbb{T})\right.$ is invertible $\}$, then the kernel $\ell$ of the above contraction is one-dimensional and it is spanned by the vector $\left.\Gamma\right|_{(q, v, t)}$; the subspace $\ell$ is called the null-space of $\Omega$

- whenever $\mathbb{Q}$ is potential-generated $\left\{\Leftrightarrow \mathbb{Q}_{i}=-\frac{\partial \mathbb{U}}{\partial q^{i}}+\frac{\mathrm{d}}{\mathrm{d} t}\left(\frac{\partial \mathbb{U}}{\partial v^{i}}\right)\right\}$, $\theta_{\mathbb{L}}:=\mathrm{d} t+\left(\partial_{v^{i}} \mathbb{L}\right)\left\{\mathrm{d} q^{i}-v^{i} \mathrm{~d} t\right\}$

is the Lepage one-form on the extended tangent bundle that is associated to the Lagrangian $\mathbb{L}=\mathbb{T}-\mathbb{U}$.

- the two-form $\Omega$ and also the one-form $\theta_{\mathbb{L}}$ are invariant with respect to the group of diffeomorphisms of the "space-time" $M \times \mathbb{R}$, which are of the form:

$\left(q^{i}, t\right) \mapsto\left(Q^{i}=Q^{i}(q, t), T=t\right)$

So we can claim: Lagrangian mechanics is determined by the null-spaces of the distinguished two-form $\Omega$. Finding them, it is enough to pick up at each null subspace $\ell$ a vector $w$ for which $w \downarrow \mathrm{d} t=1$. Doing this, we are point-wisely reconstructing the dynamical vector field (4). Its integral curves are solutions of the Lagrange equations.

\section{Variational principle and "umbilical strings"}

Suppose there are given Newton-Lagrange type equations of motion with forces of any origin:

$\dot{q}^{i}=v^{i}, \dot{v}^{i}=F^{i}(q, v, t) \Leftrightarrow$ kernel of $\Omega$

(made of the ingredients $\mathbb{T}=\frac{1}{2} \delta_{a b} v^{a} v^{b}$ and $\mathbb{Q}_{i}=F^{i}$ )

In what follows we will provide a variational principle for the above set of differential equations just in terms of the distinguished two-form $\Omega$ introduced above. Variation will be carried over bit peculiar objects, namely the surfaces in the extended tangent bundle.

Down-to-earth, let us fix

- two points in the extended configuration space $M \times \mathbb{R}$, initial and final events $\left(q_{0}, t_{0}\right)$ and $\left(q_{1}, t_{1}\right)$

- any extended tangent bundle curve

$\gamma_{\mathrm{ref}}: \tau \in\left\langle t_{0}, t_{1}\right\rangle \mapsto(q=q(\tau), v=v(\tau), t=\tau) \in T M \times \mathbb{R}$, such that $q\left(t_{0}\right)=q_{0}$ and $q\left(t_{1}\right)=q_{1}$

The space of admissible "umbilical surfaces" of the reference curve $\gamma_{\text {ref }}$ is defined as follows:

$\mathrm{Umb}\left(\gamma_{\mathrm{ref}}\right):=\left\{\Sigma:(\tau, \sigma) \in\left\langle t_{0}, t_{1}\right\rangle \times\langle 0,1\rangle \mapsto \Sigma(\tau, \sigma)\right.$

$=(q=q(\tau, \sigma), v=v(\tau, \sigma), t=\tau) \in T M \times \mathbb{R}$,

such that for all values

of the parameter $\sigma: q\left(\tau=t_{0}, \sigma\right)=q_{0}, q\left(\tau=t_{1}, \sigma\right)=q_{1}$

of the parameter $\left.\tau: \Sigma(\tau, \sigma=0)=\gamma_{\text {ref }}(\tau)\right\}$.

Here $\tau \in\left\langle t_{0}, t_{1}\right\rangle$ is the time parameter, $\sigma$ is some "worldsheet" distance coordinate from the unit interval and the second edge curve $\gamma(\tau):=\Sigma(\tau, \sigma=1)$, see Fig. 2.

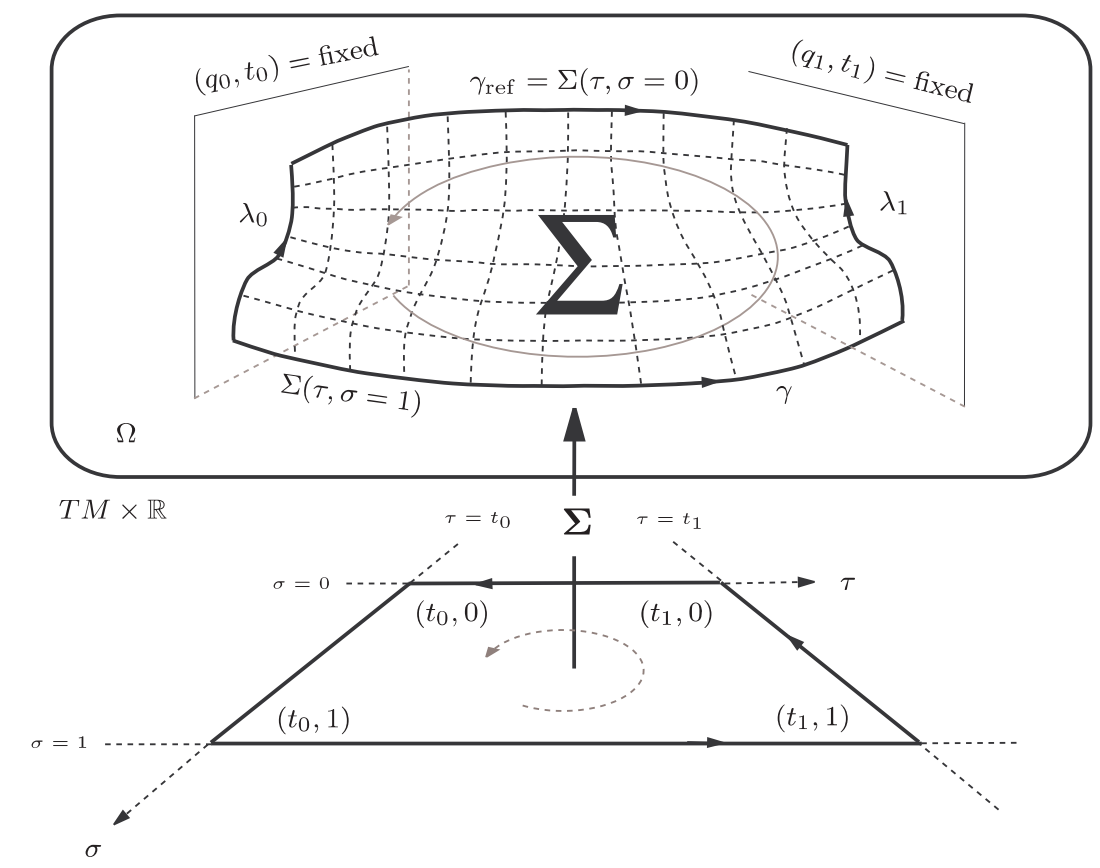

Fig. 2: Oriented "umbilical surface" $\Sigma$ connects the reference curve $\gamma_{\text {ref }}$ with the "history" $\gamma(\tau)=\Sigma(\tau, \sigma=1)$. Sideways boundary curves $\lambda_{0}(\sigma)=\Sigma\left(t_{0}, \sigma\right)$ and $\lambda_{1}(\sigma)=\Sigma\left(t_{1}, \sigma\right)$ are located within the $n$-dimensional submanifolds $\left(q_{0}, t_{0}\right)=$ fixed and $\left(q_{1}, t_{1}\right)=$ fixed of the extended tangent bundle. In the figure, these edge submanifolds are schematically represented by two-dimensional "D-branes." 
In the above class of all admissible "umbilical world-sheets" a stationary surface of the action

$$
S: \Sigma \mapsto S(\Sigma):=\int_{\Sigma} \Omega
$$

satisfies the following two equations:

$$
\begin{aligned}
& \text { boundary term: } \left.0=\frac{\mathrm{d}}{\mathrm{d} \tau} \gamma,\right\lrcorner \Omega, \\
& \text { bulk term: } 0=\mathrm{d} \Omega\left(\frac{\partial}{\partial \tau} \Sigma, \frac{\partial}{\partial \sigma} \Sigma, \cdot\right) .
\end{aligned}
$$

The first equation states that tangent vector $\frac{\mathrm{d}}{\mathrm{d} \tau} \gamma$ lies in the kernel of $\Omega$. This means that the second edge $\Sigma(\tau, \sigma=1)$ of the sought stationary surface satisfies the initial Newton-Lagrange differential system (6). By its definition, it should be a classical trajectory $\gamma_{\text {class }}$ that connects the space-time events $\left(q_{0}, t_{0}\right)$ and $\left(q_{1}, t_{1}\right)$. The genuineness of $\gamma_{\text {class }}=\Sigma(\tau, \sigma=1)$ is obvious, and it does not depend on the chosen auxiliary reference curve $\gamma_{\text {ref }}$.

The complete solution of the variational problem provides as a bonus also some stationary (also called minimal) surface $\Sigma_{\text {min. }}$. It is anchored to the curves $\gamma_{\text {ref }}$ and $\gamma_{\text {class }}$ and is trapped in between the "D-brane type" submanifolds $\left(q_{0}, t_{0}\right)=$ fixed and $\left(q_{1}, t_{1}\right)=$ fixed in the extended tangent bundle. Whether such a stationary "umbilical" surface exists depends on properties of the physical system under consideration.

\section{Quantization: path versus surface integral}

In the case when classical dynamics (6) is "derivable" from the Lepage one-form $\theta_{\mathbb{L}}$, i.e. $\Omega=\mathrm{d} \theta_{\mathbb{L}}$, one can use for quantization the Feynman prescription [11, 12]. According to Feynman, the probability amplitude of the transition of the system from the space-time configuration $\left(q_{0}, t_{0}\right)$ to $\left(q_{1}, t_{1}\right)$ is given as follows:

$\mathbf{A}\left(q_{0}, t_{0}, q_{1}, t_{1}\right) \propto \int_{\mathcal{G}}[\mathcal{D} \gamma] \exp \left\{\frac{i}{\hbar} \int_{\gamma} \theta_{\mathbb{L}}\right\}$.

The "path-summation" here is taken over the class $\mathcal{G}$ of all admissible curves in $T M \times \mathbb{R}$, as drawn in Fig. 3. The exponent in (10) is the standard curve integral of the one-form $\theta_{\mathbb{L}}$ over $\gamma$. The questions of the measure $[\mathcal{D} \gamma]$ and the proper normalization of the probability amplitude are discussed in the next section.

We have already noted that classical mechanics is only $\Omega$ sensitive. On the other hand the sensitivity of quantum mechanics on its one-form potential precursor $\theta_{\mathbb{L}}$ is ultimately evident from the Feynman prescription. In what follows, we propose some modifications to (10), leading to the replacement of $\theta_{\mathbb{L}}$ by the two-form $\Omega$. This will enable us to "quantize" dissipative systems as well.

Our main trick is a simple rearrangement based on the Stokes theorem. Down-to-earth, in the class $\mathcal{G}$ that enters the "path-summation" in (10), there is one specially distinguished curve, the classical trajectory. Using it, for any other $\gamma$ within this class we get an oriented loop (cycle):

$$
\partial \Sigma:=\gamma+\lambda_{1}-\gamma_{\text {class }}-\lambda_{0} .
$$

Here $\lambda_{0}$ and $\lambda_{1}$ are arbitrarily chosen curves within the "D-branes" $\left(q_{0}, t_{0}\right)=$ fixed and $\left(q_{1}, t_{1}\right)=$ fixed, see Fig. 2 . The restriction of $\theta_{\mathbb{L}}$ to any of these edge submanifolds is trivial, therefore we can write:

$\int_{\gamma} \theta_{\mathbb{L}}-\int_{\gamma_{\text {class }}} \theta_{\mathbb{L}}+\underbrace{\int_{\lambda_{1}} \theta_{\mathbb{L}}-\int_{\lambda_{0}} \theta_{\mathbb{L}}}_{\text {zero }}=\int_{\partial \Sigma} \theta_{\mathbb{L}}=\int_{\Sigma} \mathrm{d} \theta_{\mathbb{L}}$,

where $\Sigma \in \operatorname{Umb}\left(\gamma_{\text {class }}\right)$.

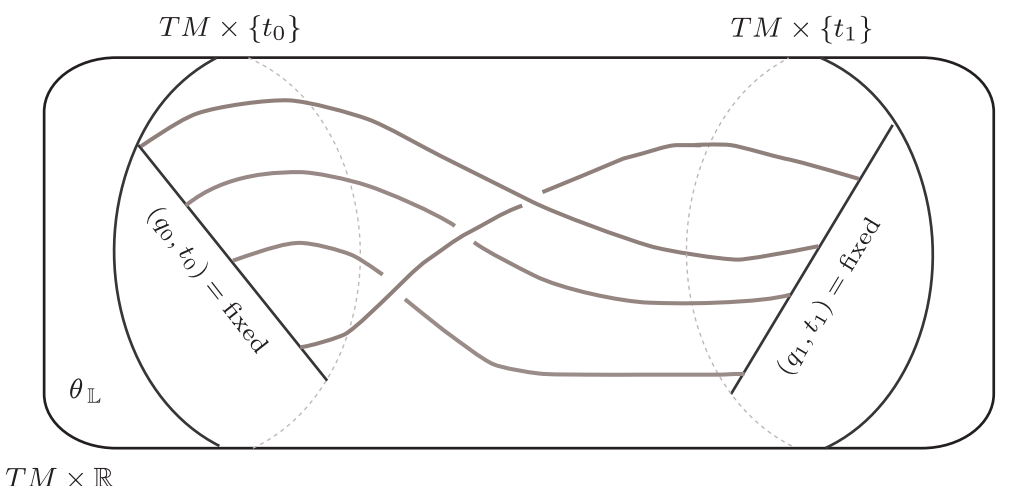

$\mathbb{R}$

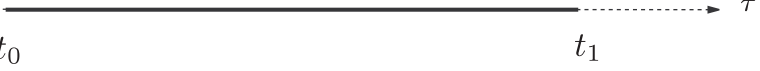

Fig. 3: Schematic picture of the class $\mathcal{G}=\left\{\gamma: \tau \mapsto(q=q(\tau), v=v(\tau), t=\tau)\right.$, such that $q\left(t_{0}\right)=q_{0}$ and $\left.q\left(t_{1}\right)=q_{1}\right\}$ 
Let us remind the reader that

- the integral of $\theta_{\mathbb{L}}$ over the curve $\gamma_{\text {class }}$ gives the value of the classical action $S_{\text {class }}$

- the existence of the "umbilical" string $\Sigma$ that connects $\gamma$ and $\gamma_{\text {class }}$ is determined by the topological (homological) properties of the extended tangent bundle

- define $\mathrm{Vol}_{\gamma}$ as the "number" of surfaces in $\mathrm{Umb}\left(\gamma_{\text {class }}\right)$ containing $\gamma$ and $\gamma_{\text {class }}$ as the subboundaries, assuming that all elements of $\mathcal{G}$ are homotopically equivalent, $\operatorname{Vol}_{\gamma}$ becomes $\gamma$-independent

Motivated by the trick (11) and assuming no topological obstructions on the side of $T M \times \mathbb{R}$ we can slightly rearrange the transition amplitude (10) as follows:

$$
\begin{aligned}
\mathbf{A}\left(q_{0}, t_{0} ; q_{1}, t_{1}\right) \propto & \exp \left\{\frac{i}{\hbar} S_{\text {class }}\right\} \\
& \int_{\mathcal{G}}[\mathcal{D} \gamma] \frac{\operatorname{Vol}_{\gamma}}{\operatorname{Vol}_{\gamma}} \exp \left\{\frac{i}{\hbar}\left(\int_{\gamma}-\int_{\gamma_{\text {class }}}\right) \theta_{\mathbb{L}}\right\} \\
\propto & \frac{1}{\operatorname{Vol}_{\gamma}} \exp \left\{\frac{i}{\hbar} S_{\text {class }}\right\} \\
& \int_{\mathcal{G}}\{[\mathcal{D} \gamma] \operatorname{Vol} \gamma\} \exp \left\{\frac{i}{\hbar} \int_{\partial \Sigma} \theta_{\mathbb{L}}\right\} \\
\propto & \exp \left\{\frac{i}{\hbar} S_{\text {class }}\right\} \\
& \int_{\operatorname{Umb}}[\mathcal{D} \Sigma] \exp \left\{\frac{i}{\hbar} \int_{\Sigma} \mathrm{d} \theta_{\mathbb{L}}\right\},
\end{aligned}
$$

Here in the last line we have included the numerical prefactor $\mathrm{Vol}_{\gamma}^{-1}$ to normalization and, because the integrand is only boundary dependent, the path integration was extended to the surface integration over the umbilical class $\mathrm{Umb} \equiv \mathrm{Umb}\left(\gamma_{\text {class }}\right)$.

From formulas (12) and (13) we get the transition probability amplitude in the product form of the classic phase and quantum corrections. In general, the role of $d \theta_{\mathbb{L}}$ is played by the distinguished two-form $\Omega$ and therefore we can presume to express the contribution of the quantum corrections in any case (including dissipativity) as follows:

$$
\text { Q.C. } \propto \int_{\text {Umb }}[\mathcal{D} \Sigma] \exp \left\{\frac{i}{\hbar} \int_{\Sigma} \Omega\right\} \text {. }
$$

If we also have a suitable candidate for the classical phase, we will be able to write:

$$
\begin{aligned}
\mathbf{A}\left(q_{0}, t_{0} ; q_{1}, t_{1}\right) & \propto \text { C.P. } \times \text { Q.C. } \\
& =\exp \left\{\frac{i}{\hbar} S_{\text {class }}^{\Omega}\right\} \int_{\mathrm{Umb}}[\mathcal{D} \Sigma] \exp \left\{\frac{i}{\hbar} \int_{\Sigma} \Omega\right\} .
\end{aligned}
$$

In my two previous papers $[5,6]$ I proposed for C.P. the following procedure: first find the classical solution $\gamma_{\text {class }}$ of the problem (6), then split the forces into the potential-generated and the non-potential-generated parts, i.e.

$$
\mathbb{Q}_{i}=\left\{-\partial_{q^{i}} \mathbb{U}+\frac{\mathrm{d}}{\mathrm{d} t}\left(\partial_{v^{i}} \mathbb{U}\right)\right\}+\mathbb{Q}_{i}^{\text {rest }},
$$

at third introduce subsidiary-like Lagrangian $\mathbb{T}-\mathbb{U}$ using the potential $\mathbb{U}$ and express the C.P. term in the form:

$$
\text { C.P. }=\exp \left\{\frac{i}{\hbar} S_{\text {class }}^{\Omega}\right\}=\exp \left\{\frac{i}{\hbar} \int_{\gamma_{\text {class }}} \theta_{\mathbb{T}-\mathbb{U}}\right\} .
$$

This procedure, however, seems not to be absolutely correct. Lack of the classical limit becomes more-or-less apparent here (see the results in the next section). When varying of the above term we do not get back the initial differential system (6). The classical limit, or equivalently, the classical time evolution of the expectation values of the corresponding physical operators (Ehrenfest theorem) is achieved only in the regime where $\mathbb{Q}_{i}^{\text {rest }}$ can be treated as small perturbations to potential-generated forces. Thus, (15) with the C.P. term of the form (16) can serve as a type of effective perturbation theory when the perturbations are not potential-generated.

\section{An example: quantum mechanics with friction}

Let us focus on the perturbation-like quantization of the dynamics of a unit mass particle moving in $M=\mathbb{R}[x]$, which is driven by the conservative force $\mathbb{F}=-\frac{\mathrm{d}}{\mathrm{d} x} \mathbb{U}(x)$ along with the friction $\mathbb{Q}^{\text {rest }}=-\kappa v$. The extended tangent bundle $T \mathbb{R}[x, v] \times \mathbb{R}[t]$ corresponds to an ordinary three-dimensional Cartesian space. The distinguished two-form takes the simple form

$\Omega=-\kappa v \mathrm{~d} x \wedge \mathrm{d} t+\mathrm{d}\left\{v \mathrm{~d} x-\left\{\frac{1}{2} v^{2}+\mathbb{U}(x)\right\} \mathrm{d} t\right\}$.

Our aim is to evaluate the transition amplitude as a function of the initial and final events. Suppose we have chosen a solution $\gamma_{\text {class }}(\tau)=\left(x_{\text {class }}(\tau), v_{\text {class }}(\tau)=\dot{x}_{\text {class }}(\tau), t=\tau\right)$ of the Newton-Lagrange equation of motion:

$\ddot{x}=\mathbb{F}(x)-\kappa \dot{x}$, which respect the initial and final conditions: $x_{\text {class }}\left(t_{0}\right)=q_{0}$ and $x_{\text {class }}\left(t_{1}\right)=q_{1}$.

Direct application of formula (15) then leads to the following expression:

$$
\begin{aligned}
& \mathbf{A}\left(q_{0}, t_{0} ; q_{1}, t_{1}\right) \propto \exp \left\{\frac{i}{\hbar} S_{\text {class }}\right\} \\
& \int_{\mathrm{Umb}}[\mathcal{D} \Sigma] \exp \left\{\frac{i}{\hbar}\left\{\int_{\partial \Sigma}\left\{v \mathrm{~d} x-\left\{\frac{1}{2} v^{2}+\mathbb{U}(x)\right\} \mathrm{d} t\right\}-\kappa \int_{\Sigma} v \mathrm{~d} x \wedge \mathrm{d} t\right\}\right\} .
\end{aligned}
$$




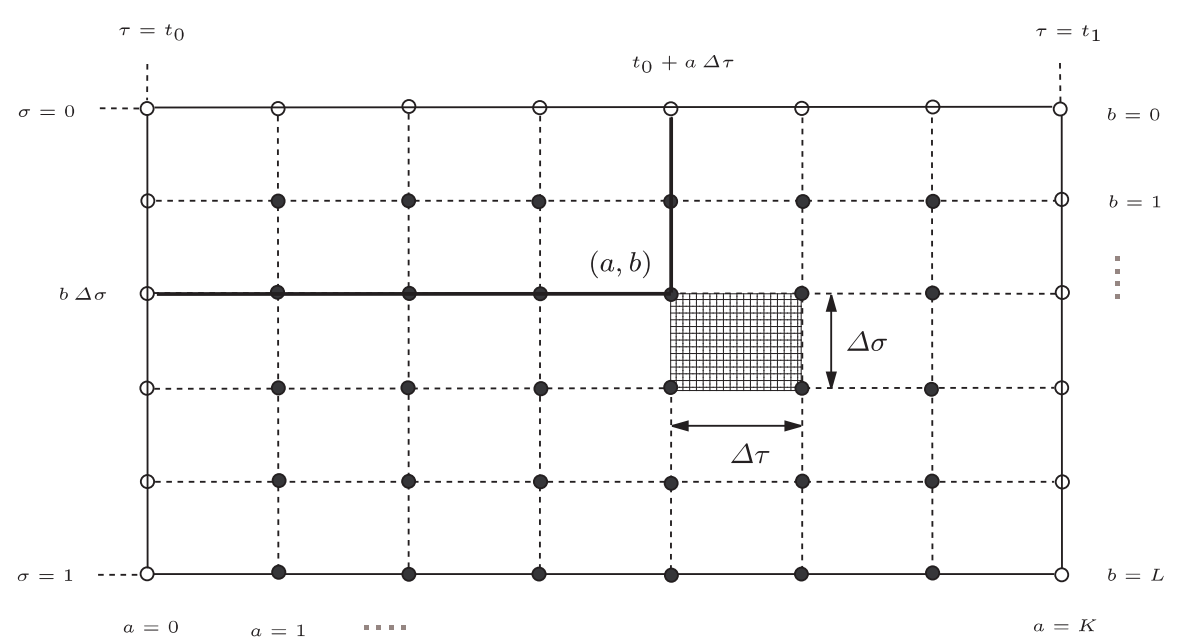

Fig. 4: Schematic picture of the nodal grid, points marked with empty circles are constrained by (18).

To evaluate the world-sheet functional integral entering the above formula, let us introduce a grid in the underlying parametric space $\left\langle t_{0}, t_{1}\right\rangle \times\langle 0,1\rangle \subset \mathbb{R}^{2}[\tau, \sigma]$ :

$$
\{(a, b)\}_{\text {nodes }}:=\left\{\left(t_{0}+a \Delta \tau, b \Delta \sigma\right) \in \mathbb{R}^{2}[\tau, \sigma] ;\right.
$$

time index $a$ runs from 0 to $K$ and distance index $b$ runs from 0 to $L$ \}

We get rectangular tiles, each of which encloses the infinitesimal area $\Delta \tau \Delta \sigma=\frac{t_{1}-t_{0}}{K} \cdot \frac{1-0}{L}$. At the end the numbers $K$ and $L$ will be sent to infinity and the volume $\Delta \tau \Delta \sigma \rightarrow \mathrm{d} \tau \mathrm{d} \sigma$.

After this splitting has been performed, any "umbilical" string

$$
\Sigma: \mathbb{R}^{2}[\tau, \sigma] \rightarrow \mathbb{R}^{3}[x, v, t], \quad(\tau, \sigma) \mapsto \Sigma(\tau, \sigma)=(x(\tau, \sigma), v(\tau, \sigma), t(\tau, \sigma)=\tau)
$$

can be discretized by evaluating its coordinate functions at the nodes of the considered grid $\{(a, b)\}_{\text {nodes }}$, i.e.

$$
\Sigma:\{(a, b)\}_{\text {nodes }} \rightarrow\left\{\Sigma(a, b)=\left(x_{(a, b)}, v_{(a, b)}, t_{0}+a \Delta \tau\right)\right\}
$$

Here, to keep the ensemble $\Sigma(a, b) \subset \mathbb{R}^{3}[x, v, t]$ within the considered "umbilical" class $\mathrm{Umb} \equiv \mathrm{Umb}\left(\gamma_{\text {class }}\right)$, we must impose:

$$
\begin{cases}\forall a=0, \ldots, K & \left\{x_{(a, 0)}=x_{\text {class }}\left(t_{0}+a \Delta \tau\right), x_{(a, 0)}=x_{\text {class }}\left(t_{0}+a \Delta \tau\right)\right\} \Leftrightarrow\left\{\Sigma(\tau, \sigma=0)=\gamma_{\text {class }}(\tau)\right\} \\ \forall b=1, \ldots, L & \left\{x_{(0, b)}=q_{0}, x_{(K, b)}=q_{1}\right\} \Leftrightarrow\left\{\Sigma\left(\tau=t_{0}, \sigma\right) \text { and } \Sigma\left(\tau=t_{1}, \sigma\right) \subset \text { "D - branes" }\right\}\end{cases}
$$

Therefore, formally, the functional integral over all possible string configurations is a formal limit of the ordinary integrals taking them over all unconstrained variables in the ensemble $\{\Sigma(a, b)\}$, i.e.:

$$
\int_{\mathrm{Umb}}[\mathcal{D} \Sigma]:=\lim _{\substack{K \rightarrow \infty \\ L \rightarrow \infty}}\left\{\int_{-\infty}^{\infty} \ldots \int_{-\infty}^{\infty} \prod_{a=1}^{K-1} \prod_{b=1}^{L} \mathrm{~d} x_{(a, b)} \mathrm{d} v_{(a, b)} \mathrm{d} v_{(0, b)} \mathrm{d} v_{(K, b)}\right\}
$$

When step-wisely discretizing the integrals in the exponent of (17), taking into account the constraints (18), we get for the bulk term (everything is done with respect to the chosen orientation of the "umbilical" world-sheet $\Sigma$ ):

$$
\begin{aligned}
\int_{\Sigma} v \mathrm{~d} x \wedge \mathrm{d} t & =\int_{t_{0}}^{t_{1}} \mathrm{~d} \tau \int_{0}^{1} \mathrm{~d} \sigma\left\{v(\tau, \sigma) \frac{\partial x}{\partial \sigma}(\tau, \sigma)\right\}=\lim _{\substack{K \rightarrow \infty \\
L \rightarrow \infty}} \sum_{a=0}^{K-1} \sum_{b=0}^{L-1} \Delta \tau \Delta \sigma\left\{v_{(a, b)} \frac{x_{(a, b+1)}-x_{(a, b)}}{\Delta \sigma}\right\} \\
& =\lim _{\substack{K \rightarrow \infty \\
L \rightarrow \infty}}\left\{\sum_{a=1}^{K-1} \sum_{b=1}^{L-1} \Delta \tau v_{(a, b)}\left\{x_{(a, b+1)}-x_{(a, b)}\right\}+\sum_{a=0}^{K-1} \Delta \tau v_{(a, 0)} x_{(a, 1)}\right\}-\int_{t_{0}}^{t_{1}} \mathrm{~d} \tau\left\{v_{\text {class }}(\tau) x_{\text {class }}(\tau)\right\}
\end{aligned}
$$

and similarly for the boundary term:

$$
\begin{aligned}
& \int_{\partial \Sigma}\left\{v \mathrm{~d} x-\left\{\frac{1}{2} v^{2}+\mathbb{U}(x)\right\} \mathrm{d} t\right\}=\int_{\Sigma(\tau, \sigma=1)}\left\{v \mathrm{~d} x-\left\{\frac{1}{2} v^{2}+\mathbb{U}(x)\right\} \mathrm{d} t\right\}-\int_{\Sigma(\tau, \sigma=0)}\left\{v \mathrm{~d} x-\left\{\frac{1}{2} v^{2}+\mathbb{U}(x)\right\} \mathrm{d} t\right\} \\
& =\lim _{K \rightarrow \infty} \sum_{a=0}^{K-1}\left\{v_{(a, L)}\left\{x_{(a+1, L)}-x_{(a, L)}\right\}-\Delta \tau\left\{\frac{1}{2} v_{(a, L)}^{2}+\mathbb{U}\left(x_{(a, L)}\right)\right\}\right\}-\int_{t_{0}} \mathrm{~d} \tau\left\{\frac{1}{2}\left(v_{\text {class }}(\tau)\right)^{2}+\mathbb{U}\left(x_{\text {class }}(\tau)\right)\right\} .
\end{aligned}
$$


Putting everything together, integrating over all variables apart subensemble $\left\{x_{(a, L)}\right\}$ and returning back to the continuum limit, we get the following expression for the transition amplitude:

$$
\mathbf{A}\left(q_{0}, t_{0} ; q_{1}, t_{1}\right) \propto \exp \left\{\frac{i}{\hbar} \int_{t_{0}}^{t_{1}} \mathrm{~d} \tau\left\{\kappa v_{\text {class }} x_{\text {class }}\right\}\right\}[\mathcal{D} x(\tau)] \exp \left\{\frac{i}{\hbar} \int_{t_{0}}^{t_{1}} \mathrm{~d} \tau\left\{\frac{1}{2} \dot{x}^{2}-\mathbb{U}(x)-\kappa x v_{\text {class }}\right\}\right\} .
$$

The phase factor in front of (19) comes from the definition of the classical action $S_{\text {class }}^{\Omega}$ and from the world-sheet functional integration. The second term is the standard Feynman path integral, which is taken over the histories $\{\tau \mapsto(x(\tau):=x(\tau, \sigma=1), t=\tau)\}$ in the extended configuration space $\mathbb{R}[x] \times \mathbb{R}[t]$. However, in comparison with the standard formula, a new term appears here. It is an external source generated by the classical velocity $v_{\text {class }}$.

Let us remind the reader how to treat ugly infinite constants emerging in the functional integration. If the entering infinities are functionally independent of the coordinates of space-time events, then one can easily neglect them. The only important term inside the functional integral is the phase factor, which depends on the coordinates of $\left(q_{0}, t_{0}\right)$ and $\left(q_{1}, t_{1}\right)$, i.e. we need to seize the following quantity:

$$
\mathbf{A}\left(q_{0}, t_{0} ; q_{1}, t_{1}\right) \propto \exp \left\{\frac{i}{\hbar} \text { Phase }\left(q_{0}, t_{0} ; q_{1}, t_{1}\right)\right\},
$$

anything else is just an inherited rudiment. The proper normalization of the amplitude $\mathbf{A}\left(q_{0}, t_{0} ; q_{1}, t_{1}\right)$ is dictated by its physical meaning. The square of its absolute value answers the question about the probability density of observing a particle in a sufficiently small neighborhood of the configuration $\left(q_{1}, t_{1}\right)$, when before it was observed in a neighborhood of the space-time position $\left(q_{0}, t_{0}\right)$. This implies the desired normalization conditions (since we are dealing with the space-time continuum, the normalization to a $\delta$-function should be employed):

$$
\begin{aligned}
& \quad\left\{t_{1} \rightarrow t_{0} \Rightarrow \mathbf{A}\left(q_{0}, t_{0} ; q_{1}, t_{1}\right) \rightarrow \delta\left(q_{1}-q_{0}\right)\right\} \Leftrightarrow\left\{\text { at time } t_{0} \text { the system occupies definite position } q_{0}\right\} \\
& \int_{-\infty}^{\infty} \mathrm{d} q_{1} \mathbf{A}\left(q_{0}, t_{0} ; q_{1}, t_{1}\right) \mathbf{A}^{*}\left(q_{0}^{\prime}, t_{0} ; q_{1}, t_{1}\right)=\delta\left(q_{0}^{\prime}-q_{0}\right) \Leftrightarrow\{\text { conservation of the total probability }\}
\end{aligned}
$$

Having everything at hand, let us compute the normalized probability amplitude with the presence of friction in the cases when $\mathbb{U}(x)=0$ (free particle) and $\mathbb{U}(x)=\frac{1}{2} \omega x^{2}$ (linear harmonic oscillator).

\subsection{Free particle with damping}

Putting together all fragments that enter formula (19) and taking into account the required normalization conditions, we arrive at the following probability amplitude:

$$
\begin{aligned}
& \mathbf{A}\left(q_{0}, t_{0} ; q_{1}, t_{1}\right)=\frac{1}{\sqrt{2 \pi i \hbar \lambda}} \exp \left\{\frac{i}{2 \hbar \lambda}\left(q_{1}-q_{0}\right)^{2}\right\}, \\
& \text { where } \quad \lambda=\frac{2}{\kappa} \tanh \left\{\frac{\kappa}{2}\left(t_{1}-t_{0}\right)\right\} .
\end{aligned}
$$

A short inspection of (20) discloses that if $\kappa \rightarrow 0$, then $\lambda \rightarrow\left(t_{1}-t_{0}\right)$ and the above amplitude $\mathbf{A}\left(q_{0}, t_{0} ; q_{1}, t_{1}\right)$ coincides with the ordinary quantum propagator for a free particle.

Let us perform an analysis of the time evolution in terms of the transition probability amplitude (20) From the point of view of quantum mechanics, the best fit of a unit mass particle with the classical initial condition $\left(q_{0}=0, v_{0}=v_{0}, t_{0}=0\right)$ is the Gaussian wave-packet

$$
\Psi(x) \propto \exp \left\{-\frac{x^{2}}{2 \xi^{2}}+\frac{i}{\hbar} x v_{0}\right\}
$$

with some initial width $\xi$. At a later time $t$, the system under consideration will be characterized by the convoluted wave-packet distribution

$$
\Psi(x, t) \propto \int_{-\infty}^{\infty} \mathrm{d} q \Psi(q) \mathbf{A}(q, 0 ; x, t) .
$$

It preserves its Gaussian shape, and its main characteristics, the mean value of the position $\langle x\rangle$ and the actual width of the wave-packet $\Xi^{2}$, vary with time according to

$$
\langle x\rangle=v_{0} \lambda \text { and } \Xi^{2}=\xi^{2}+\frac{\hbar^{2}}{\xi^{2}} \lambda^{2} .
$$

The velocity of the center of the wave-packet $\frac{\mathrm{d}}{\mathrm{d} t}\langle x\rangle=\frac{4 v_{0} \mathrm{e}^{-\kappa t}}{\left(1+\mathrm{e}^{-\kappa t}\right)^{2}} \propto v_{0} \mathrm{e}^{-\kappa t}$, i.e. it decreases for $t \gg 1$ exponentially, as one would predict on classical intuition.

\subsection{Damped harmonic oscillator}

The probability amplitude for a damped harmonic oscillator with unit mass requires a solution of Newton's equation $\ddot{x}=-\omega^{2} x-\kappa \dot{x}:$

$$
x_{\text {class }}(\tau)=\mathrm{e}^{-\frac{\kappa}{2} \tau}\left\{A \mathrm{e}^{i \Theta \tau}+B \mathrm{e}^{-i \Theta \tau}\right\},
$$

where the new frequency $\Theta=\sqrt{\omega^{2}-\frac{\kappa^{2}}{4}}$.

When substituting the general $\mathbb{U}(x)$ in (19) by the oscillator potential $\frac{1}{2} w^{2} x^{2}$ and doing simple algebraic manipulations, we arrive at: 


$$
\begin{aligned}
& \mathbf{A}\left(q_{0}, t_{0} ; q_{1}, t_{1}\right)=\mathbb{K}\left(t_{1}-t_{0}\right) \\
& \exp \left\{\frac { i } { \hbar } \frac { \Theta } { 2 \operatorname { s i n } \{ \Theta ( t _ { 1 } - t _ { 0 } ) \} } \left[\left(q_{1}^{2}+q_{0}^{2}\right) \cos \left\{\Theta\left(t_{1}-t_{0}\right)\right\}\right.\right. \\
& \left.\left.\quad-2\left(q_{1} q_{0}\right) \operatorname{ch}\left\{\kappa / 2\left(t_{1}-t_{0}\right)\right\}\right]\right\},
\end{aligned}
$$

where the normalization factor

$$
\mathbb{K}\left(t_{1}-t_{0}\right)=\sqrt{\frac{\Theta \operatorname{ch}\left\{\frac{\kappa}{2}\left(t_{1}-t_{0}\right)\right\}}{2 \pi i \hbar \sin \left\{\Theta\left(t_{1}-t_{0}\right)\right\}}} .
$$

It is clear that

- taking the limit $\omega \rightarrow 0$; the above amplitude reproduces the free particle result (20),

- in the limit $\kappa \rightarrow 0$; the Schrödinger propagator for a harmonic oscillator with frequency $\omega$ is recovered.

\section{Acknowledgment}

Many thanks (in alphabetical order) go to Vladimír Balek, Pavel Bóna, Marián Fecko, Tamás Fülöp, Peter Prešnajder, Artur Sergyeyev and Pavol Ševera for their interest, criticism, fruitful discussions and many useful comments. This research was supported in part by Comenius University Grant UK/359/2006, VEGA Grant 1/3042/06 and ESF project JPD3 BA-2005/1-034.

\section{References}

[1] Dyson, F. J.: Feynman's Proof of the Maxwell Equations, Am. J. Phys. Vol. 58 (1990), p. 209-211.

[2] Henneaux, M.: Equations of Motion, Commutation Relations and Ambiguities in the Lagrangian Formalism, Annals Phys. Vol. 140 (1982), p. 45-64.

[3] Sarlet, W.: The Helmholtz Condition Revisited. A New Approach to the Inverse Problem of Lagrangian
Dynamics, J. Phys. A: Math. Gen. Vol. 15 (1982), p. 1503-1517.

[4] Henneaux, M.: On the Inverse Problem of the Calculus of Variations, J. Phys. A: Math. Gen. Vol. 15 (1982), p. L93-L96.

[5] Kochan, D.: How to Quantize Forces (?): An Academic Essay How the Strings Could Enter Classical Mechanics, submitted to JMP, hep-th/0612115.

[6] Kochan, D.: Direct Quantization of Equations of Motion: from Classical Dynamics to Transition Amplitudes via Strings, submitted to CMP, hep-th/0703073.

[7] Arnol'd, V. I.: Mathematical Methods of Classical Mechanics ( $2^{\text {nd }}$ ed.), Springer-Verlag, 1989.

[8] Abraham, R., Marsden, J. E.: Foundation of Mechanics, Addison-Wesley, 1978.

[9] Burke, W. L.: Applied Differential Geometry, Cambridge University Press, 1985.

[10] Fecko, M.: Differential Geometry and Lie Groups for Physicists, Cambridge University Press, 2006.

[11] Feynman, R. P., Hibbs, A. R.: Quantum Mechanics and Path Integral, McGraw-Hill, 1965.

[12] Faddeev, L. D., Slavnov, A. A.: Gauge Fields: An Introduction to Quantum Theory (2 ${ }^{\text {nd }}$ ed.), Addison-Wesley, 1991.

Mgr. Denis Kochan, Ph.D.

phone: +4210260295460

e-mail:kochan@fmph.uniba.sk

Department of Theoretical Physics and Didactics of Physics

Comenius University of Bratislava

Faculty of Matematics Physics and Iinformatics

Mlynská dolina F2

84248 Bratislava, Slovakia 